\title{
Non-contrast MR portography using time-spatial labeling inversion pulse for diagnosis of portal vein pathology
}

\author{
Amr Ahmed Mubarak, Ghada Elsaed Awad, Mohamed Adel Eltomey* and Mahmoud Abd Elaziz Dawoud
}

\begin{abstract}
Background: To study the ability of non-contrast MR portography using time-spatial labeling inversion pulse (T-SLIP) as a non-invasive contrast-free imaging modality to delineate different portal vein pathological conditions. The study included 25 patients with known history of portal vein disease and another 25 age-matched patients with normal portal vein. Both groups were examined by respiratory-triggered non-contrast MR portography using time-spatial labeling inversion pulse technique. Image quality was assessed first, and findings of diagnostic scans were compared to color duplex ultrasonography and selectively in those with diseased portal vein to portal-phase images of dynamic contrast-enhanced MRI.

Results: Significant relation was found between breathing regularity and image quality in T-SLIP sequence, with diagnostic scans sensitivity and specificity of $89.29 \%$ and $86.21 \%$, respectively, for diagnosis of different portal vein pathological conditions.

Conclusions: Non-contrast MR portography is a useful technique for diagnosis of portal vein pathology in carefully selected patients.
\end{abstract}

Keywords: Portal vein, Portography, Non-contrast, Time-spatial, T-SLIP, Magnetic resonance

\section{Background}

Accurate radiological assessment of portal vein is crucial to rule out portal vein disease, to detect anatomical variations, and to draw a road map for surgeons planning for hepatectomy or liver transplant [1].

Portal vein assessment has been traditionally carried out using different imaging modalities including color duplex ultrasonography, contrast-enhanced computed tomography $(\mathrm{CT})$, and contrast-enhanced magnetic resonance imaging (MRI) as well as different diagnostic invasive angiographic techniques including percutaneous transhepatic portography and splenoportography [2]. Each of these techniques has certain drawbacks: the operator-dependent nature of ultrasound and its suboptimal performance in patients with poor acoustic window, the risk of ionizing radiation and contrast-induced nephropathy and allergic reactions in $\mathrm{CT}$, and the risk of

\footnotetext{
* Correspondence: mohamed.eltomey@med.tanta.edu.eg

Radiology and Imaging Department, Faculty of Medicine, Tanta University, Medical Complex, Elgiesh (Elbahr) Street, Tanta, Egypt
}

nephrogenic systemic fibrosis with gadolinium chelates used in contrast-enhanced MRI [3, 4]. Moreover, the accurate timing of contrast bolus together with competent long breath holds are mandatory to obtain good quality contrasted-enhanced MR portography images, which are not possible all the time due to technologist error or poor patient compliance [5].

Non-contrast MR portography using time-spatial labeling inversion pulse (T-SLIP) technique has emerged as a non-invasive contrast-free imaging tool with promising preliminary results regarding image quality. It is based on respiratory-triggered $3 \mathrm{D}$ acquisition using time-spatial labeling inversion pulse combined with either true steady-state free precession or half-Fourier fast spin echo (FSE) pulse sequences [6].

Two different methods have been implemented using this technique for non-contrast T-SLIP MR portography, the flow-in and flow-out methods. In the flow-in method, selective inversion recovery tagging pulse is used for suppression of blood signal when it is placed on the vessels of no interest like aorta and inferior vena cava, giving a black 
blood signal, allowing the untagged fresh blood within the portal vein to appear as a bright blood signal. The flowout method uses both a non-selective inversion recovery pulse and a selective inversion recovery tagging pulse. First, the non-selective inversion pulse inverts all magnetization in the region to suppress background signal, followed by a spatially selective tagging pulse placed over extrahepatic portal vein to selectively restore the magnetization in the tagged region to its original value, depicting the tagged blood as a bright blood signal [7].

A modified T-SLIP technique has been introduced recently using compressed sensing acceleration-enabled non-contrast MR portography to be performed in a single breath hold with acceptable image quality [8].

To the best of our knowledge, non-contrast MR portography technique using T-SLIP sequence was used on healthy volunteers and not on patients having portal vein pathology. So, the study was aimed at testing the performance of this technique in delineation of different portal vein pathological conditions.

\section{Methods \\ Study population}

A case control study was conducted prospectively on two groups of patients; the first one included 25 patients known to have different portal vein pathological conditions whereas the second group included age-matched 25 patients with no previous history of portal vein disease to act as a control group. Both groups were examined first by color duplex ultrasonography to document portal vein status followed by non-contrast MR portography using time-spatial labeling inversion pulse technique. The diseased group underwent dynamic contrastenhanced MRI of the liver as a part of their routine imaging work-up. The study duration was 1 year starting from January 2018 to December 2018.

Informed consent was obtained from individuals participating in this study after explanation of the benefits and possible risks of the procedure. The participants were screened carefully to exclude those with contraindication to MRI examination. The study was approved by our local institutional ethical committee.

\section{Color duplex ultrasonography}

All participants in this study underwent color duplex ultrasonography of the portal vein before non-contrast MR portography exam, using ultrasound machine Aplio 500 (Canon Medical Systems) equipped with $3.5 \mathrm{MHz}$ convex array transducer. Portal vein size, patency, peak systolic velocity, and flow direction were documented. Only patients with satisfactory visualization of the portal vein and its pathology were included in the study.

\section{Non-contrast MR portography technique}

All participants lied supine on MRI table with arms raised above head and their feet entering the magnet bore first. Respiratory bellows was placed around the upper abdomen to acquire respiratory-triggered sequences with meticulous care to ensure it yields a good respiratory monitoring trace on scanner screen. A phased array surface body coil was placed on the lower chest and upper abdomen. Reassurance and brief explanation of the procedure were done to alleviate anxiety and to ensure participant's cooperation. IV cannula was placed for those who underwent contrast-enhanced dynamic MRI study.

\section{Scanning protocol}

All studies were performed using 1.5 T MRI scanner (Vantage Titan, Canon Medical Systems). After 3-plane localizers, axial and coronal half-Fourier T2 images with breath hold were obtained to delineate the liver and portal vein. Parameters are as follows: axial images (TR $=14,700$ $\mathrm{ms}, \mathrm{TE}=120 \mathrm{~ms}$, matrix $=192 \times 256, \mathrm{FOV}=38 \times 42 \mathrm{~cm}$, acceleration factor $=2)$ and coronal images $(\mathrm{TR}=9500$ $\mathrm{ms}, \mathrm{TE}=120 \mathrm{~ms}$, matrix $=224 \times 256, \mathrm{FOV}=35 \times 40 \mathrm{~cm}$, acceleration factor $=2$ ). Non-contrast $M R$ portography sequence was acquired in coronal plane using respiratorytriggered $3 \mathrm{D}$ balanced steady-state free precession (b-SSFP) sequence combined with time-spatial labeling inversion pulse with flow-out technique. The obtained axial and coronal T2 images were used to plan the non-contrast MR portography sequence; axial images were used to ensure adequate number of slices to cover portal vein and its intrahepatic branches while coronal images were used for placement of the tagging inversion pulse which was placed obliquely parallel to the inferior liver edge. Additional saturation pulse was applied superiorly above the liver to block the inflow effect of aorta, and another saturation pulse was placed inferiorly in oblique orientation to block the inflow effect of inferior vena cava without blocking the inflow effect of superior mesenteric vein which contributes to portal vein flow (Fig. 1). Acquisition parameters were as follows: $\mathrm{TR}=5.2, \mathrm{TE}=2.6 \mathrm{~ms}, \mathrm{FOV}=40 \times 37 \mathrm{~cm}$, matrix size $=256 \times 256$, and slice thickness $=3 \mathrm{~mm}$, and black blood time interval (BBTI) was set at $1200 \mathrm{~ms}$. Scan time was variable according to the number of slices acquired and respiratory rate of the participant.

\section{Image post-processing}

The obtained coronal source images were processed on MRI scanner console to generate 3D maximum intensity projection images (MIP) and colored $3 \mathrm{D}$ volumerendered images in different planes after image clipping and manual removal of undesirable structures. 


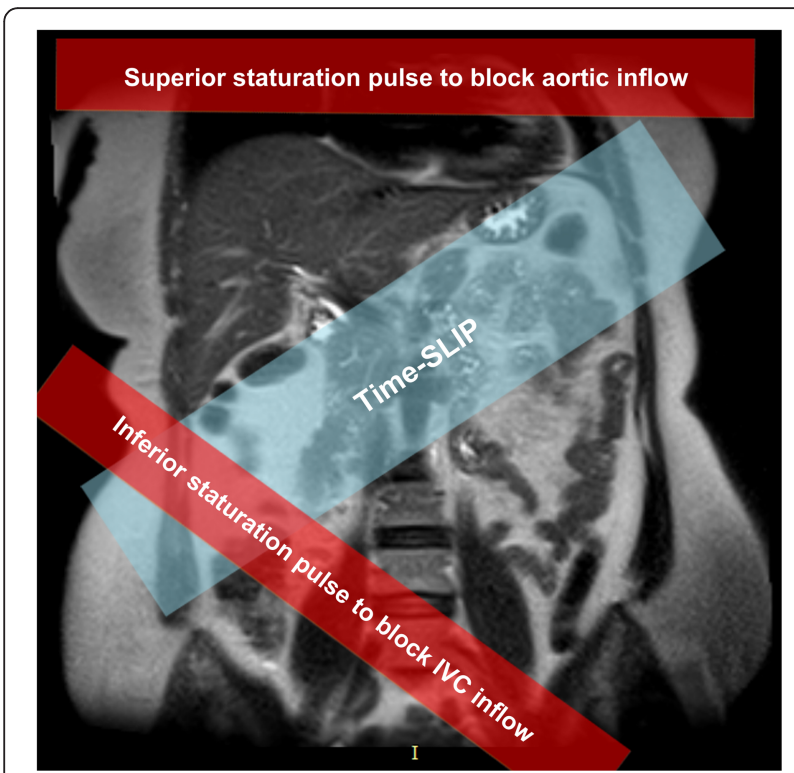

Fig. 1 Planning of non-contrast MR portography. Placement of timespatial labeling inversion pulse (T-SLIP) parallel to inferior liver edge (blue) with additional superior and inferior saturation pulses (reds) to block aortic and IVC inflows, respectively

\section{Dynamic contrast-enhanced MRI protocol}

All 25 participants with known portal vein pathology underwent contrast-enhanced dynamic MRI of the liver, and $3 \mathrm{D}$ gradient-echo T1-weighted fat-suppressed axial images $\left(\mathrm{TR}=4.8 \mathrm{~ms}, \mathrm{TE}=1.9 \mathrm{~ms}, \quad\right.$ flip angle $=15^{\circ}$, matrix $=192 \times 256, \quad$ FOV $=40 \times 35$, slice thickness $=7$ $\mathrm{mm}$, and scan time $=13-16 \mathrm{~s}$ ) were acquired with breath holding after manual IV injection of gadolinium-based contrast agent, gadopentetate dimeglumine (Magnevist, Bayer Healthcare), at a dose of $0.1 \mathrm{mmol} / \mathrm{kg}$ followed by $10 \mathrm{cc}$ saline flushing. The arrival of contrast material at abdominal aorta was detected by real-time bolus tracking built-in software (Visual prep). Actual MR acquisition started $8 \mathrm{~s}$ after contrast detection at abdominal aorta to acquire late arterial phase. Portal-venous phase was acquired $10 \mathrm{~s}$ after late arterial one, and delayed phase acquired 2 min thereafter.

\section{Image analysis of non-contrast MR portography Assessment of image quality}

Non-contrast MR portography studies were assessed in conjoint sessions by three radiologist with total experience of 15 years in interpreting abdominal MRI studies; they were aware of duplex ultrasound results before interpreting images. Images were assessed first for image quality by scrolling through source images to detect respiratory and voluntary motion artifacts, to assess the degree of portal circulation flow-related enhancement, and to rule out the presence of any intra-abdominal fluid material like ascites, fluid-filled stomach, distended GB, or biliary radicals that may interfere with adequate visualization of portal vein at MIP reconstructions. Accordingly, each study was assigned a quality score as follows: (1) good quality scan-absent respiratory and voluntary motion artifacts, adequate flow-related enhancement at portal vein or its tributaries, and absent fluid-filled material obscuring portal vein at MIP images; (2) moderate quality scan-presence of mild to moderate respiratory/voluntary motion artifacts, adequate flowrelated enhancement at portal vein or its tributaries, and/or presence of fluid material obscuring portal vein at MIP images; and (3) poor quality non-diagnostic scan-presence of severe respiratory/voluntary motion artifacts and/or inadequate flow-related enhancement at portal vein or its tributaries. Good and moderate quality scans were considered interpretable and were grouped together when performing statistical analysis. The noninterpretable scans were discarded.

\section{Assessment of portal vein}

Good and moderate quality scans were selected for portal vein assessment by analysis of reconstructed MIP and volume-rendered images, and scrolling through coronal source images. The presence of intraluminal filling defect, eccentric or concentric narrowing, luminal dilatation, and cavernous transformation were reported. Any associated dilatation of splenic vein and coronary vein or the presence of varices was reported as well. All findings of non-contrast MR portography were compared to findings of color duplex ultrasonography as a reference. Only patients with good delineation of the portal vein and/or its pathology on color Doppler ultrasound were included in the study. In addition, the findings of diseased portal vein at non-contrast MR portography images were also compared to portal-phase dynamic contrast-enhanced MRI images.

\section{Statistical analysis}

Two sample $t$ test for equal means was used for comparison between the groups, and chi-square test was used for testing relationships between categorical variables. Calculation of sensitivity, specificity, and accuracy of T-SLIP MR portography was also done. All tests were performed on Minitab 17 statistical software package (Minitab Inc.).

\section{Results}

The study included 25 patients: 14 males and 11 females, known to have different portal vein pathological conditions with their age ranging from 20 to 70 years with a mean of $58.1 \pm 11.9 \mathrm{SD}$. The study also included agematched 25 individuals not known to have portal vein pathology to act as a control group.

Non-contrast MR portography was performed completely at both control and diseased patients without the 
Table 1 Image quality analysis of T-SLIP non-contrast MR portography

\begin{tabular}{llllll}
\hline Scan quality & \multicolumn{2}{l}{ Diseased group } & & \multicolumn{2}{c}{ Control group } \\
\cline { 2 - 3 } & No. & & & No. & $\%$ \\
\hline Good quality & 8 & 32 & & 16 & 64 \\
Moderate quality & 11 & 44 & & 9 & 36 \\
Poor quality & 6 & 24 & & 0 & 0 \\
Total & 25 & 100 & & 25 & 100 \\
\hline
\end{tabular}

need for scan termination. The scan time of T-SLIP sequence ranged from $5 \mathrm{~min}$ and $49 \mathrm{~s}$ to $9 \mathrm{~min}$ and $50 \mathrm{~s}$ with a mean of $8 \mathrm{~min}$ and $7 \mathrm{~s}$ with no statistically significant difference in scan length between both groups.

As regards the image quality of non-contrast MR portography studies, Tables 1 and 2 show the analysis of image quality among the studied groups. The control group showed 16 scans with good quality and 9 scans with moderate quality; 6 of them showed mild to moderate respiratory motion artifacts as a consequence of irregular breathing, and 3 of them showed dilated biliary radicals. On the other hand, the group with portal vein disease showed 8 scans with good quality, 11 scans with moderate quality, and 6 scans with poor quality. All scans with moderate quality showed mild to moderate respiratory motion artifacts with example presented in Fig. 2, ascites was present at three patients, and dilated intrahepatic biliary radicals were present at two patients. As regards poor quality scans, four scans showed severe respiratory and voluntary motion artifacts, two scans showed poor flow-related enhancement of portal circulation, and three patients had ascites.

In both studied groups, 44 patients had good or moderate quality scans and 6 showed poor quality scans with 24 patients showing regular breathing and 26 showing irregular breathing. Analysis of the relation between breathing regularity and image quality using chi-square test showed a statistically significant relation with $p$ value $<0.0001$.

In both studied groups, six patients had ascites, three of them showed poor quality scans while the other three showed scans of moderate quality. Dilated intrahepatic biliary radicals were seen in five patients who showed moderate quality of their images. Analysis of the relation between ascites and image quality using chi-square test did not show a statistically significant relation with $p$ value $=0.290$. No relation was found between the presence of dilated intrahepatic biliary radicals and image quality with $p$ value $=0.749$.

Assessment of portal vein flow-related enhancement at T-SLIP sequence showed good delineation of the vein in 21 out of 25 (84\%) of the control cases and 19 out of the $25(76 \%)$ cases with known portal vein pathology. However, three of the later cases showed an apparently normal portal vein despite being abnormal at duplex ultrasonography and contrast-enhanced MRI. The overall sensitivity and specificity for the T-SLIP non-contrast MR portography were $89.29 \%$ and $86.21 \%$, respectively; the test accuracy was $87.72 \%$.

Different portal vein pathologies were diagnosed by $\mathrm{T}$ SLIP sequence in the studied 25 patients with examples presented in Figs. 3, 4, and 5, including 15 patients with portal vein thrombosis (60\%) either partial or complete, 5 patients with portal hypertension (20\%), 4 patients with cavernous transformation of the portal vein (16\%), and 1 patient who showed post-left hepatectomy occlusion of the main portal vein (4\%). As regards portal vein anatomy, it was clearly delineated in diagnostic scans with one anatomical variant encountered in this study where portal vein trifurcation was present (Fig. 6).

\section{Discussion}

Time-spatial labeling inversion pulse had been incorporated into different pulse sequences to image different vascular structures including renal and carotid arteries without the use of exogenous contrast agents $[9,10]$. The same MRI technique showed promising results for noncontrast imaging of portal vein as well. Non-contrast MR portography initially depended on flow-in T-SLIP technique using either b-SSPF sequence or half-Fourier FSE sequence, with introduction of flow-out method later on which showed promising results as well for portal vein imaging [7]. In this study, we used the flow-out method and respiratory-triggered 3D b-SSFP sequence combined with T-SLIP to image the portal vein in patients harboring diseased portal vein and in the control group.

The average scan time for T-SLIP non-contrast MR portography in this study was $8 \mathrm{~min}$ and $7 \mathrm{~s}$; this time is longer than that stated by Shimada et al. [6] who reported a mean scan time of $2 \mathrm{~min}$ and $40 \mathrm{~s}$. After revising the scan parameters they used, the longer scan times in this

Table 2 Distribution of image quality score items among the studied groups

\begin{tabular}{|c|c|c|c|c|c|c|}
\hline & \multicolumn{2}{|c|}{ Breathing } & \multicolumn{2}{|l|}{ Ascites } & \multicolumn{2}{|c|}{ Dilated biliary radicals } \\
\hline & Regular & Irregular & Present & $\overline{\text { Absent }}$ & Present & Absent \\
\hline Diseased group & 8 & 17 & 6 & 19 & 2 & 23 \\
\hline Control group & 16 & 9 & 0 & 25 & 3 & 22 \\
\hline Chi-square* & \multicolumn{2}{|c|}{$p$ value $<0.0001$} & \multicolumn{2}{|c|}{$p$ value $=0.290$} & \multicolumn{2}{|c|}{$p$ value $=0.749$} \\
\hline
\end{tabular}

${ }^{*}$ Chi-square test was done to study the relation between image quality and three items of image quality score including breathing regularity, presence of ascites, and intrahepatic biliary radicals. $p$ value was considered significant if $<0.0001$ 


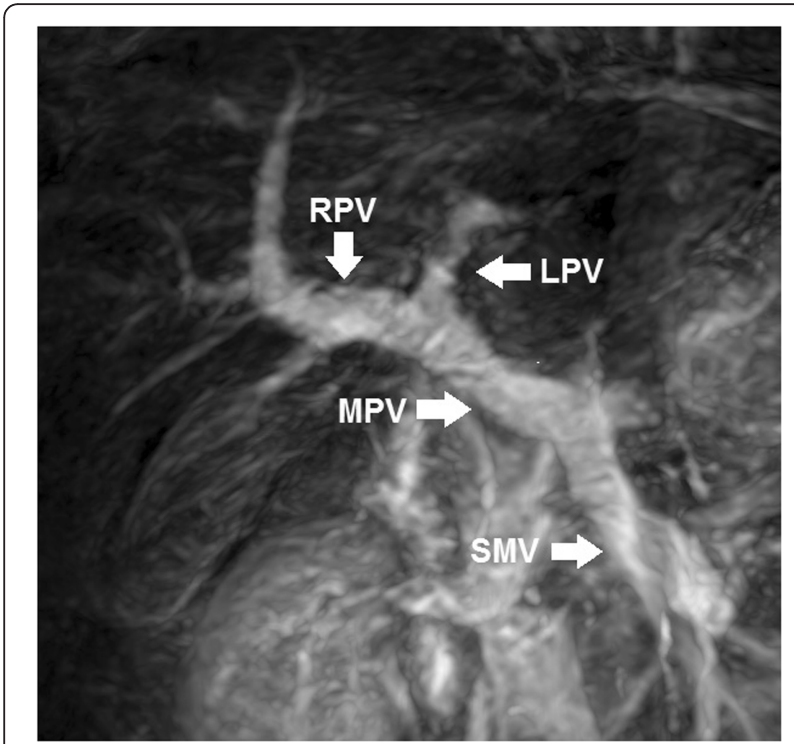

Fig. 2 Moderate quality scan. Coronal maximum intensity projection image from a non-contrast MR portography using T-SLIP technique showing respiratory motion artifacts due to patient's irregular breathing. However, main portal vein and its branches are interpretable and appear to be patent which was confirmed by color duplex ultrasonography

study are probably attributed to longer TR and increased number of slices. The long scan time has the drawback of being difficult for patients, three in this study, to remain still and breathe regularly during entire acquisition due to the presence of significant amount of ascites with resultant non-diagnostic poor quality scans. It is noteworthy that a different imaging technique was presented by Ito et al. [11] with shorter acquisition time who used a single breath-hold ECG-synchronized acquisition with promising results but at a lower spatial resolution.
In this study, a significant relation was found between the regularity of breathing and the image quality which could be explained by the principle of the sequence being a respiratory-triggered one; regular breathing pattern is essential during the entire acquisition time for obtaining interpretable scans necessitating well-fitted respiratory bellows or optimal setup of navigator scan and adequate patient training [12]. Six non-diagnostic scans were encountered in this study due to irregular breathing in non-cooperative patients and in those with significant amount of ascites interfering with normal diaphragmatic movements.

This study showed no significant relation between portal vein visualization and the presence of ascites or biliary radical dilatation. Even when they were present, we used coronal source images to evaluate portal vein clearly. Yoshikawa et al. [13] stated that the use of b-SSFP sequence combined with T-SLIP to visualize portal vein could be problematic in patients with dilated intrahepatic and extrahepatic bile ducts as well as in those with significant amount of ascites or huge porta-hepatis cystic lesions. They relied on the fact that this pulse sequence is fluid sensitive and would project such abnormal fluids over portal vein at MIP images interfering with portal vein assessment. The difference between our results and their statement could be attributed to the use of coronal source images in addition to MIP images when evaluating portal vein and the amount of ascites and degree of bile ducts dilatation encountered in this study.

In this study, portal vein anatomy delineation was excellent in both the control and diseased groups with portal vein trifurcation variant presented in one patient. As regards portal vein pathology, the overall performance of non-contrast MR portography at diagnostic quality scans for detection of portal vein pathological conditions was compared to results obtained from color duplex

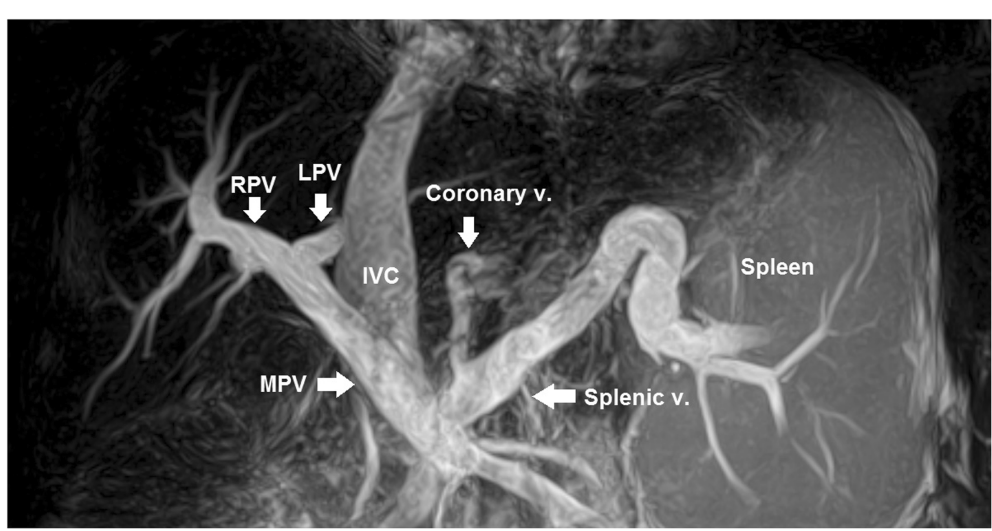

Fig. 3 Portal hypertension. Coronal maximum intensity projection image from a non-contrast MR portography using T-SLIP technique showing dilated portal and splenic veins with sizeable patent coronary vein denoting portal hypertension. Splenomegaly is noted as well. The inevitable flow-related enhancement of intrahepatic segment of IVC obscures the part of left portal branch which requires further post-processing for removal. MPV, main portal vein; RPV, right portal vein; LPV, left portal vein; IVC, inferior vena cava 

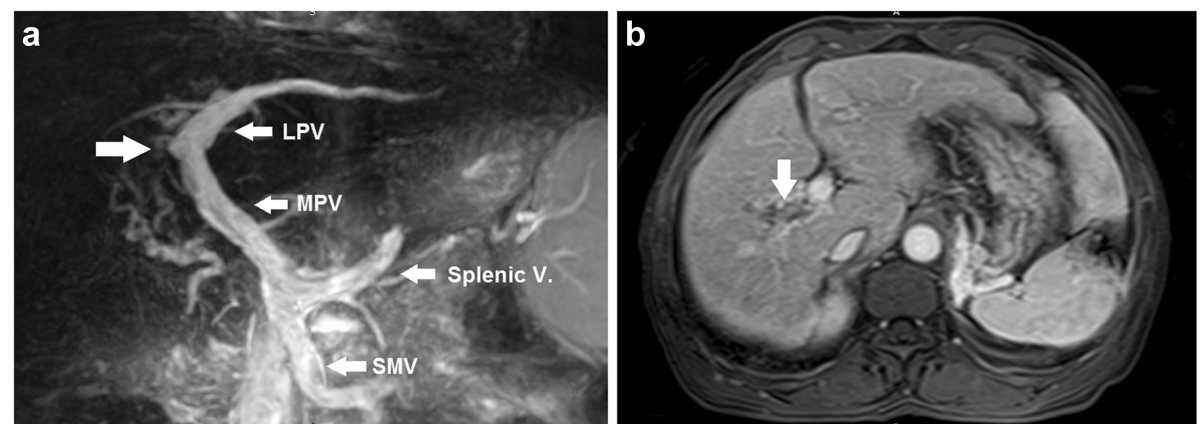

Fig. 4 Right portal vein occlusion. a Coronal MIP image from a non-contrast MR portography using T-SLIP sequence showing total occlusion of right portal branch (arrow) with eccentric filling defect seen at the distal end of the main portal vein representing partial thrombosis. b Axial 3D gradient-echo fat-suppressed T1-weighted contrast-enhanced MR image during portal phase showing hypointense filling defect at the right portal branch (arrow) confirming right portal branch occlusion. MPV, main portal vein; LPV, left portal vein; SMV, superior mesenteric vein

ultrasonography and contrast-enhanced MR portography for the same patients. The overall sensitivity and specificity for the T-SLIP non-contrast MR portography were $89.29 \%$ and $86.21 \%$, respectively; the test accuracy was $87.72 \%$.

To the best of our knowledge, the use of T-SLIP sequence in the assessment of portal vein was applied for normal individuals only. Comparison of our results to others could not be achieved; however, during the course of the study, we were able to point some potential problems and adjustments in the application of the sequence as well as the selection of patients. This technique was introduced for selective visualization of portal vein. However, different arterial and venous vascular structures of no interest may appear during scan requiring technique modification for the suppression of their signals [6].

In our study, we applied an oblique inferior saturation pulse at both common iliac veins to block the inflow
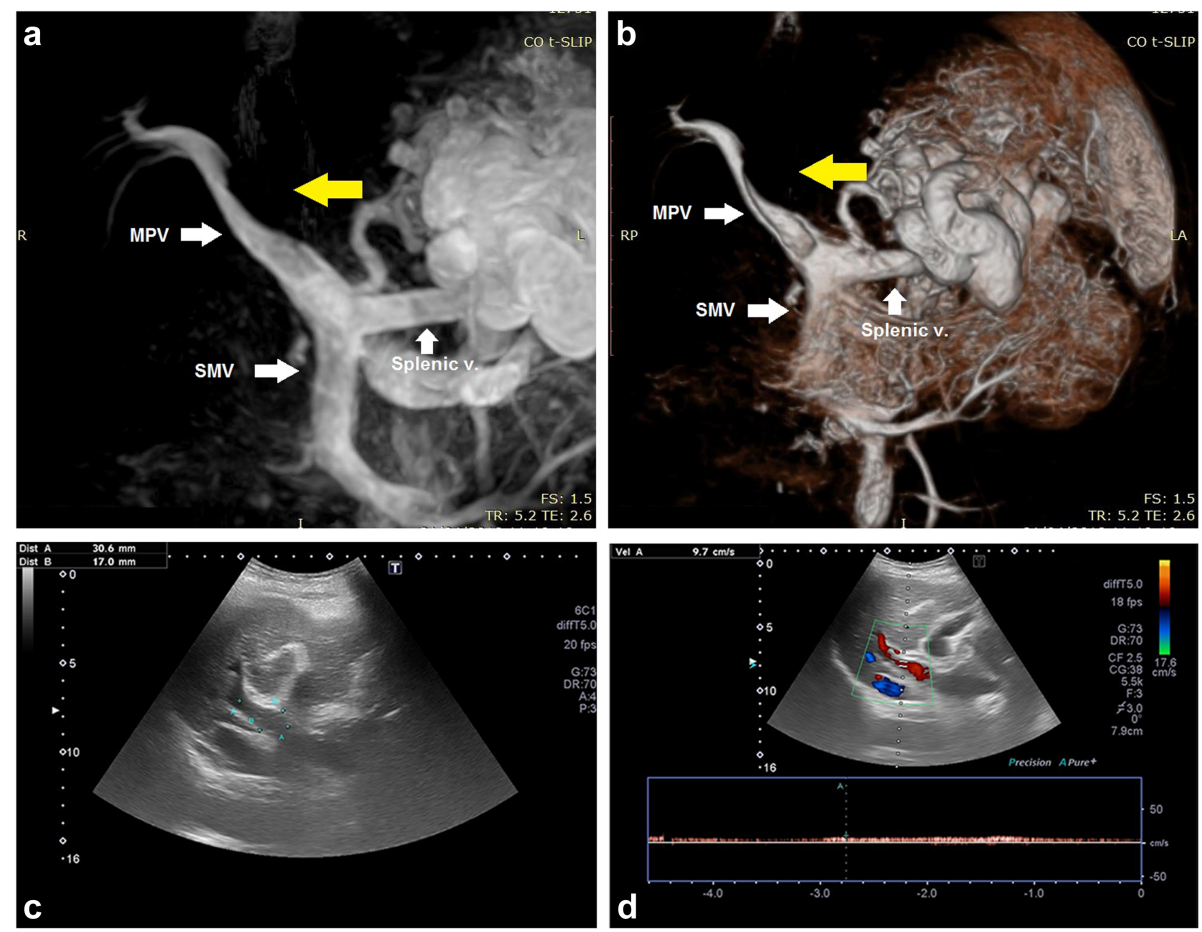

Fig. 5 Partial thrombosis of main portal vein. Coronal MIP image (a) and 3D volume-rendered image (b) from a non-contrast MR portography using T-SLIP sequence showing eccentric mural narrowing of the main portal vein (yellow arrow) representing partial thrombosis. Greyscale ultrasound image of the same patient (c) shows echogenic material within main portal vein, with partial color saturation and Doppler flow detected at color duplex ultrasound image (d) denoting partial thrombosis confirming non-contrast MR portography finding. MPV, main portal vein; SMV, superior mesenteric vein 


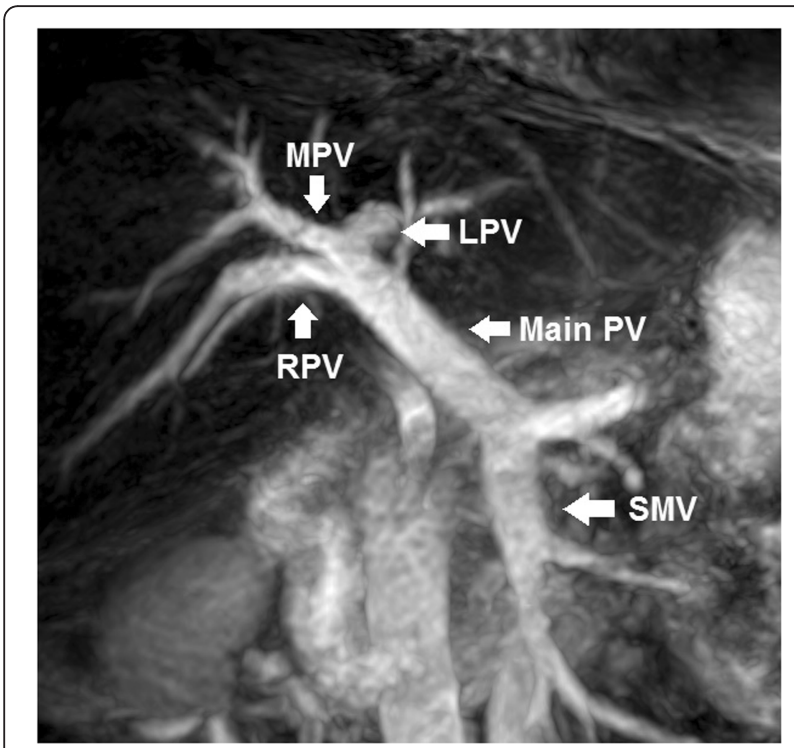

Fig. 6 Portal vein trifurcation. Coronal maximum intensity projection image from a non-contrast MR portography using T-SLIP technique showing anatomical variant where the main portal vein trifurcates into the right portal vein (RPV), middle portal vein (MPV), and left portal vein (LPV)

effect of inferior vena cava (IVC); most of our studies showed flow-related enhancement at the intrahepatic segment of IVC due to the presence of non-saturated venous flow which is coming from both renal veins. The application of inferior saturation pulse at a higher level opposite renal hila would certainly eliminate the intrahepatic IVC inflow effect, but would also block the inflow effect of superior mesenteric vein which contributes to portal vein flow. The non-evitable flow-related enhancement at the intrahepatic segment of IVC may interfere with the visualization of left portal vein at MIP images (Fig. 3) necessitating further relatively time-consuming post-processing to remove it. Such inevitable IVC inflow effect was previously encountered by Shimada et al. [6, 14 in their studies which was problematic in the assessment of portal vein at MIP images, and they had to look at source images instead to examine portal vein.

Another observation in this study was that we used a fixed value of black blood time interval (BBTI): $1200 \mathrm{~ms}$ in both control group and patients with diseased portal vein. This may explain why the portal vein in two of the diseased individuals was not visualized. The use of higher BBTIs in those patients would have probably allowed better visualization of intrahepatic branches of portal vein. Moreover, we only used flow-out method combined with b-SSPF pulse sequence. The use of flow-in method and half-Fourier FSE sequence would also have allowed for a more detailed study of the technique. All of the previous scan parameter modifications would certainly come at the expense of prolonged scan time, leading to patient discomfort, so we could not be able to apply them, keeping in mind that the initial T-SLIP sequence scan time is around $8 \mathrm{~min}$ not including other sequences.

Limitations of this study include the small number of subjects and the lack of similar published articles testing the use of this sequence on diseased portal veins. Further studies with different BBTIs and different pulse sequences are also required to define the optimum BBTI for each portal vein pathological entity and to optimize image quality in patients with diseased portal vein.

\section{Conclusions}

In conclusion, based on our findings non-contrast MR portography using time-spatial labeling inversion pulse is considered a promising, helpful non-invasive contrastfree diagnostic tool for demonstrating different portal vein pathological conditions with diagnostic scans obtained in carefully selected patients who breathe regularly and tolerate long scan times.

\section{Abbreviations}

BBTI: Black blood time interval; CT: Computed tomography; FSE: Fast spin echo; MR: Magnetic resonance; T-SLIP: Time-spatial labeling inversion pulse

\section{Acknowledgements}

Not applicable

\section{Authors' contributions}

All authors have appraised the article and actively contributed in the work. AM contributed to the idea of the research, writing of initial manuscript, and imaging and adjustment of MRI parameters.

GA contributed to the collection of data and follow-up of cases. ME contributed to the statistical analysis and revision of the manuscript. MD contributed to the overall revision of the manuscript. All authors have read and approved the final version of the manuscript.

\section{Funding}

The authors declare that the study was self-funded, and no funding was obtained from the manufacturer of the machine used, from any of its representatives, or from any other organization.

\section{Availability of data and materials}

The datasets generated and/or analyzed in this study are available through the corresponding author on reasonable request.

\section{Ethics approval and consent to participate}

This study was approved by the Research Ethics Committee of the Faculty of Medicine at Tanta University in Egypt on 23 December 2017 (reference number of approval: 319962/12/17). All patients included in this study gave written informed consent to participate in this research. If the patient was less than 16 years old or unconscious at the time of the study, written informed consent for their participation was given by their parent or legal guardian.

\section{Consent for publication}

All patients included in this research gave written informed consent to publish the data contained within this study. If the patient was less than 16 years old, deceased, or unconscious when consent for publication was requested, written informed consent for the publication of this data was given by their parent or legal guardian. The authors grant the publisher the consent for publication of this work.

Competing interests

The authors declare that they have no competing interests. 
Received: 2 July 2019 Accepted: 30 August 2019

Published online: 07 October 2019

References

1. Lee MW, Lee JM, Lee JY et al (2007) Preoperative evaluation of hepatic arterial and portal venous anatomy using the time resolved echo-shared MR angiographic technique in living liver donors. European Radiology 17(4): 1074-1080

2. Kreft B, Strunk H, Flacke S et al (2000) Detection of thrombosis in the portal venous system: comparison of contrast-enhanced MR angiography with intraarterial digital subtraction angiography. Radiology 216(1):86-92

3. Buhaescu I, Izzedine H (2008) Gadolinium-induced nephrotoxicity International journal of clinical practice 62(7):1113-1118

4. Heilmaier C, Sutter R, Lutz AM et al (2007) Mapping of hepatic vascular anatomy: dynamic contrast-enhanced parallel MR imaging compared with 64 detector row CT. Radiology 245(3):872-880

5. Okumura A, Watanabe Y, Dohke M et al (1999) Contrast-enhanced threedimensional MR portography. Radiographics 19(4):973-987

6. Shimada K, Isoda H, Okada T et al (2009) Non-contrast-enhanced MR portography with time-spatial labeling inversion pulses: comparison of imaging with three-dimensional half-fourier fast spin-echo and true steadystate free-precession sequences. Journal of magnetic resonance imaging : JMRI. 29(5):1140-1146

7. Furuta A, Isoda H, Yamashita R et al (2014) Non-contrast-enhanced MR portography with balanced steady-state free-precession sequence and timespatial labeling inversion pulses: comparison of imaging with flow-in and flowout methods. Journal of magnetic resonance imaging : JMRI. 40(3):583-587

8. Ono A, Arizono S, Fujimoto K et al (2017) Non-contrast-enhanced 3D MR portography within a breath-hold using compressed sensing acceleration: a prospective noninferiority study. Magnetic resonance imaging 43:42-47

9. Kurata Y, Kido A, Fujimoto K et al (2016) Optimization of non-contrastenhanced MR angiography of the renal artery with three-dimensional balanced steady-state free-precession and time-spatial labeling inversion pulse (time-SLIP) at $3 \mathrm{~T}$ MRI, in relation to age and blood velocity. Abdominal Radiology 41(1):119-126

10. Satogami N, Okada T, Koyama T et al (2009) Visualization of external carotid artery and its branches: non-contrast-enhanced MR angiography using balanced steady-state free-precession sequence and a time-spatial labeling inversion pulse. Journal of magnetic resonance imaging : JMRI 30(3):678-683

11. Ito K, Koike S, Shimizu A et al (2008) Portal venous system: evaluation with unenhanced MR angiography with a single-breath-hold ECG-synchronized 3D half-Fourier fast spin-echo sequence. AJR American journal of roentgenology 191(2):550-554

12. Ohno T, Isoda H, Furuta A et al (2015) Non-contrast-enhanced MR portography and hepatic venography with time-spatial labeling inversion pulses: comparison at 1.5 Tesla and 3 Tesla. Acta radiologica open 4(5): 2058460115584110

13. Yoshikawa T, Ohno Y, Motohara T et al (2005) Gadolinium-enhanced phasecontrast magnetic resonance portography. Magn Reson Med Sci 4(4):165-174

14. Shimada K, Isoda H, Okada T et al (2009) Unenhanced MR portography with a half-Fourier fast spin-echo sequence and time-space labeling inversion pulses: preliminary results. AJR American journal of roentgenology 193(1): 106-112

\section{Publisher's Note}

Springer Nature remains neutral with regard to jurisdictional claims in published maps and institutional affiliations.

\section{Submit your manuscript to a SpringerOpen ${ }^{\circ}$ journal and benefit from:}

- Convenient online submission

- Rigorous peer review

- Open access: articles freely available online

- High visibility within the field

- Retaining the copyright to your article

Submit your next manuscript at $\boldsymbol{\nabla}$ springeropen.com 